\title{
Conhecimento dos cirurgiões-dentistas sobre atendimento odontológico em gestantes
}

\author{
Knowledge of dental surgeons on dental care in pregnant women \\ Conocimiento de los cirujanos dentales sobre atención dental em mujeres embarazadas
}

Recebido: 05/01/2021 | Revisado: 07/01/2021 | Aceito: 09/01/2021 | Publicado: 11/01/2021

\author{
Lucas Santana Cardoso \\ ORCID: https://orcid.org/0000-0002-7673-2342 \\ Centro Universitário de João Pessoa, Brasil \\ E-mail: lucassantanac21@gmail.com \\ Bianca Maria de Melo Costa \\ ORCID: https://orcid.org/0000-0002-4825-9420 \\ Centro Universitário de João Pessoa, Brasil \\ E-mail: biancamaria_pb@hotmail.com \\ Maísa Sales Oliveira e Silva \\ ORCID: https://orcid.org/0000-0002-6321-3471 \\ Centro Universitário de João Pessoa, Brasil \\ E-mail: maisasareis0425@gmail.com \\ Tayná Marques Pessoa \\ ORCID: https://orcid.org/0000-0003-2662-1860 \\ Centro Universitário de João Pessoa, Brasil \\ E-mail: taynapessoa_@hotmail.com \\ Bianne Maria de Melo Costa \\ ORCID: https://orcid.org/0000-0002-2323-3072 \\ Centro Universitário de João Pessoa, Brasil \\ E-mail: biannemaria@hotmail.com \\ Roberta Remiliana Silva Trinta \\ ORCID: https://orcid.org/0000-0003-1868-9400 \\ Centro Universitário de João Pessoa, Brasil \\ E-mail: roberta.trinta@unipe.edu.br
}

\begin{abstract}
Resumo
$\mathrm{Na}$ cavidade bucal a cárie, gengivite e a periodontite são comumentes agravos encontrados em mulheres grávidas, precisando serem tratadas. No entanto, os cuidados com o atendimento a esse público requer muita atenção sendo resultado de muitas dúvidas de cirurgiões-dentistas. Logo, o objetivo desse estudo é descrever sobre o atendimento à gestantes na Odontologia, reunindo protocolos e avaliando se o conhecimento dos cirurgiões-dentistas são suficientes para realizar um atendimento seguro para a gestante e para o bebê. Para isso, foi realizada uma revisão narrativa da literatura que utilizou como descritores: gestantes, odontologia e odontopediatria. Foram utilizados artigos, monografias e dissertações nas bases de dados: Literatura Latino-Americana e do Caribe em Ciências da Saúde (LILACS), BBO (Bibliografia Brasileira de Odontologia), MEDLINE (National Library of Medicine) e Google Acadêmico, sendo selecionados os artigos primariamente pelo título e resumo e depois, de modo mais específico, atendendo aos critérios de inclusão e exclusão. Através da revisão pode se afirmar que as gestantes devem ter prioridade nos programas de atenção odontológica, e os cirurgiões-dentistas devem procurar desmistificar mitos e crenças em torno do atendimento. A maioria dos estudos mostrou que, apesar de muitos profissionais afirmarem sentirem-se seguros, isso não garantiu que alguns cirurgiões-dentistas respondessem corretamente alguns questionamentos. Portanto, é necessário que cada vez mais esses profissionais tornem-se aptos a prestarem esse serviço, seja através da elaboração de protocolos ou que as instituições de ensino superior abordem esse conteúdo de forma mais intensiva, pois o tratamento a gestante jamais deve ser negligenciado.
\end{abstract}

Palavras-chave: Gravidez; Odontologia; Odontopediatria.

\begin{abstract}
In the oral cavity, caries, gingivitis and periodontitis are commonly found in pregnant women and need to be treated. However, care with this audience requires a lot of attention, resulting from many doubts of dentists. Therefore, the objective of this study is to describe the assistance to pregnant women in dentistry, gathering protocols and assessing whether the knowledge of dentists is sufficient to provide safe care for pregnant women and their babies. For this, a narrative review of the literature was carried out that used as descriptors: pregnant women, dentistry and pediatric dentistry. Articles, monographs and dissertations were used in the databases: Latin American and Caribbean Literature in Health Sciences (LILACS), BBO (Brazilian Bibliography of Dentistry), MEDLINE (National Library of Medicine) and Google Scholar, being selected the articles primarily by title and abstract and then, more specifically, meeting the inclusion and exclusion criteria. Through the review, it can be stated that pregnant women should have priority in dental care programs, and dental surgeons should seek to demystify myths and beliefs surrounding care.
\end{abstract}


Most studies have shown that, although many professionals say they feel safe, this did not guarantee that some dentists would correctly answer some questions. Therefore, it is necessary that more and more these professionals become able to provide this service, either through the elaboration of protocols or that higher education institutions approach this content in a more intensive way, as the treatment for pregnant women should never be neglected.

Keywords: Pregnancy; Dentistry; Pediatric dentistry.

\section{Resumen}

En la cavidad oral, la caries, la gingivitis y la periodontitis se encuentran comúnmente en mujeres embarazadas y necesitan tratamiento. Sin embargo, el cuidado con este público requiere mucha atención, resultado de muchas dudas de los dentistas. Por tanto, el objetivo de este estudio es describir la asistencia a la gestante en odontología, recopilando protocolos y valorando si el conocimiento de los odontólogos es suficiente para brindar una atención segura a la gestante y sus bebés. Para ello, se realizó una revisión narrativa de la literatura que utilizó como descriptores: gestante, odontología y odontopediatría. Se utilizaron artículos, monografías y disertaciones en las bases de datos: Literatura Latinoamericana y del Caribe en Ciencias de la Salud (LILACS), BBO (Bibliografía Brasileña de Odontología), MEDLINE (Biblioteca Nacional de Medicina) y Google Scholar, siendo seleccionados los artículos. principalmente por título y resumen y luego, más específicamente, cumpliendo los criterios de inclusión y exclusión. A través de la revisión, se puede afirmar que las mujeres embarazadas deben tener prioridad en los programas de atención dental y los cirujanos dentistas deben buscar desmitificar los mitos y creencias que rodean la atención. La mayoría de los estudios han demostrado que, aunque muchos profesionales dicen sentirse seguros, esto no garantiza que algunos dentistas respondan correctamente algunas preguntas. Por ello, es necesario que cada vez más estos profesionales sean capaces de brindar este servicio, ya sea a través de la elaboración de protocolos o que las instituciones de educación superior aborden este contenido de una manera más intensiva, ya que nunca se debe descuidar el tratamiento a la gestante.

Palabras clave: Embarazo; Odontología; Odontología pediatrica.

\section{Introdução}

Novas descobertas sobre o impacto da vida intrauterina no futuro trouxeram grande contribuição em muitas áreas do saber, inclusive na odontologia, e compreende-se, que as ações promotoras de saúde no exercício da odontopediatria, não podem ser direcionadas apenas para as crianças, mas também para gestantes (Duque, 2013).

A manutenção e o acompanhamento de todas as fases da gestação, deve ser incluído nas atividades de rotina da Equipe de Saúde Bucal. Nenhuma necessidade de cuidados em saúde bucal das gestantes deve ser negligenciada pelo cirurgião-dentista, pelo medo de se colocar em risco a saúde do bebê. Manter o diálogo constante com o médico obstetra, aliado aos conhecimentos sobre os procedimentos seguros em cada fase da gestação, cada situação específica de gravidez dará ao cirurgião-dentista a segurança necessária para o atendimento e resolução das principais necessidades em saúde bucal das gestantes (Ribeiro, 2019).

As gestantes tornam-se pacientes de risco temporário na Odontologia devido às mudanças psicológicas, físicas e hormonais, que criam condições adversas no meio bucal. Sendo assim, elas devem ser incluídas em estudos para que o conhecimento contribua para a melhoria do atendimento deste grupo (Vasconcelos et al., 2012).

Estudos têm mostrado a recusa das pacientes em ser atendidas por cirurgiões-dentistas, essencialmente pelo medo do atendimento, em especial a alguns procedimentos, tais como as tomadas radiográficas. Assim, ressalta-se o importante papel dos profissionais da saúde, principalmente dos médicos pré-natalistas e obstetras, que mantém o vínculo principal com a gestante, no esclarecimento das dúvidas e mitos que envolvem os tratamentos odontológicos, contribuindo para sua saúde e bem estar. Em contrapartida, a conduta dos próprios cirurgiões-dentistas, durante o atendimento, reforça a insegurança das gestantes, já que muitos desses profissionais têm pouca experiência na abordagem de gestantes (Rodrigues et al., 2018).

Apesar de serem consideradas pelo Ministério da Saúde como um grupo prioritário para as ações de saúde bucal, as gestantes ainda não foram alcançadas pelos serviços públicos odontológicos na sua rotina, havendo uma baixa cobertura a essas mulheres que, devido às alterações bucais próprias desse período, necessitam de programas educativos preventivos e de um acompanhamento odontológico no pré-natal. Reforça que a atenção à saúde bucal das gestantes pode impactar 
positivamente na saúde bucal dos seus filhos, a partir de atividades preventivas e promotoras de saúde, especialmente através de educação em saúde (Nogueira, 2018).

Considerando que há muitas dúvidas por parte dos cirurgiões-dentistas sobre o atendimento odontológico em gestantes e entendendo que o profissional dessa área deve ter um aprendizado geral sobre a saúde, principalmente para repassar as orientações básicas necessárias e corretas, esse trabalho tem como objetivo descrever sobre o atendimento à gestantes na odontologia, reunindo protocolos e avaliando se os conhecimentos dos cirurgiões-dentistas são suficientes para realizar um atendimento seguro para a gestante e para o bebê.

\section{Metodologia}

Este estudo trata-se de uma revisão narrativa da literatura, qualitativa e de caráter descritivo. A revisão narrativa é caracterizada por possibilitar a aquisição e atualização de conhecimentos em um tema específico em um curto espaço de tempo, não necessitando da exigência metodologia criteriosa e são consideradas qualitativas, pois não fornecem respostas quantitativas para questões específicas (Rother, 2007). A busca de materiais foi realizada nas seguintes bases de dados: Literatura Latino-Americana e do Caribe em Ciências da Saúde (LILACS), BBO (Bibliografia Brasileira de Odontologia), MEDLINE (National Library of Medicine) e Google Acadêmico. O levantamento de dados foi realizado no mês de outubro de 2020.

Para a criação da revisão foram percorridas as seguintes etapas: definições para critérios de inclusão e exclusão; definições das informações a serem utilizadas a partir dos artigos definidos e análise dos resultados. Os critérios de inclusão foram: idioma em português acerca da temática citada e artigos na íntegra publicados no período de 2009 a 2020. Consequentemente, os critérios de exclusão são: artigos compreendidos anterior ou posteriormente ao período estabelecido e em idioma diverso ao estabelecido.

Contudo, após a busca foi feita uma leitura criteriosa de todos os artigos que foram selecionados, utilizando os critérios de inclusão citados acima, a amostra desta revisão foi constituída de 12 artigos.

\section{Resultados e Discussão}

\subsection{Atendimento odontológico na gravidez}

A gestação constitui um processo biológico natural da vida da mulher. Com tudo, nesse período ocorrem alterações fisiológicas no organismo da gestante, como alterações cardiovascular, hematológicas, respiratórias, renais, gastrointestinais e endócrinas, cujas consequências acabam refletindo na saúde bucal. Estudos demonstram a relação que existe entre a condição de saúde da cavidade oral com a saúde sistêmica, bem como as suas influências no desenvolvimento do bebê. Muitos mitos dissipados ao longo dos anos contribuem, para que mulheres nesta fase não procurem fazer o pré-natal odontológico, até mesmo em procedimentos emergenciais e, portanto, com indicação de intervenção imediata (Moretti, Garcia, Cruz, Rolim, \& Sakashita, 2017).

Além do medo e receio da gestante, existe também a insegurança por parte de muitos cirurgiões-dentistas em atender esse grupo de pacientes. Isso se dá por diversos motivos, dentre eles o início da carreira profissional, pois muitos não conseguem ter um ensino sobre esse tipo de atendimento, uma vez que são raras as universidades que possuem em sua grade curricular uma dedicação maior a gestantes, tornando uma falha na formação desses profissionais, acentuando ainda mais a sua insegurança no mercado (Assunção \& Miguel, 2015).

Estudos realizados por Cechinel el al. (2016); Rodrigues et al. (2018); Prado et al. (2019) e Bernardi, Oliveira e Masiero (2019) mostraram que a maioria dos cirurgiões-dentistas entrevistados afirmaram ter conhecimento e se sentiam 
seguros em atender pacientes grávidas. No entanto, em ambos trabalhos desenvolvidos, dúvidas e respostas incorretas foram muito relevantes por se tratar de uma paciente que se encontra em estágio de necessidades e intervenções especiais.

Caneppele, Yamamoto, Souza, Valera e Araújo (2011) e Bernardi et al. (2019), perceberam que quanto maior o tempo de formado dos participantes, menor o conhecimento sobre o atendimento odontológico à gestantes, ou seja, o tempo de experiência do profissional não resultou em maior conhecimento do assunto, ratificando assim a necessidade de atualização profissional.

No estudo de Rodrigues et al. (2018), 75,6\% dos cirurgiões-dentistas entrevistados disseram ter recebido orientações na graduação, mas esse percentual elevado não garantiu que alguns profissionais respondessem corretamente alguns questionamentos. Assim, alguns profissionais acabam negligenciando e retardando o atendimento pelo receio de comprometer a saúde do feto e pela insegurança devido aos mitos relacionados ao atendimento ontológico durante a gestação. Isso pode ser resultado de conhecimentos insuficientes e da falta de prática no atendimento a gestantes desde a graduação (Martinelli, Belotti, Poletto, Neto, \& Oliveira 2020). Dessa maneira, é importante ratificar a necessidade de capacitação e de maior interesse das faculdades em intensificar conteúdos que abordem essa temática.

\subsection{Período gestacional e sua influência}

Uma das maiores dúvidas de profissionais e gestantes é o período correto de realizar o atendimento. A maior parte dos procedimentos odontológicos em gestantes pode ser realizado em qualquer momento, porém alguns cuidados devem ser tomados como evitar sessões longas, acomodar a paciente na cadeira de modo que desconfortos sejam evitados e dar preferência a consultas em horário após a manhã, uma vez que nesse período a ânsia de vômito e risco de hipoglicemia é maior em gestantes (Bastiani et al., 2010).

No entanto, a gravidez é compreendida por muitas fases, sendo, cronologicamente, o segundo trimestre o período gestacional em que, preferencialmente, devem-se realizar os procedimentos odontológicos, por ser considerado o mais seguro (Prado et al., 2019).

Nos três primeiros meses ocorre o risco de abortos e alterações na formação do feto e no terceiro trimestre existe o perigo de provocar o parto prematuro, além dos incômodos físicos gerados pelo peso do bebê (Prado et al., 2019).

Assim, tratamentos como exodontias não complicadas, tratamentos periodontal e endodôntico, restaurações dentárias, instalação de próteses deve ser adiados, quando possíveis, para serem realizados no segundo trimestre. Mas, em casos de urgências a intervenção deve ser realizada em qualquer momento da gravidez. Já em casos de reabilitações bucais extensas e cirurgias mais invasivas devem ser postergadas para o pós-parto (Bastiani et al., 2010).

A posição das pacientes gestantes na cadeira odontológica é de extrema importância, principalmente no terceiro trimestre. À medida que o útero aumenta com o crescimento do bebê e da placenta, ele passa a ficar sobre a veia cava inferior, os vasos femorais e a aorta. A paciente não estando posicionada em decúbito dorsal para os procedimentos, o peso do útero poder aplicar pressão suficiente para impedir o fluxo sanguíneo através desses vasos principais e causar uma condição chamada hipotensão supina. Nessa condição, a pressão sanguínea cai secundariamente ao fluxo sanguíneo impedido. Essa situação é facilmente melhorada por um posicionamento adequado da gestante para o lado esquerdo e pela elevação da cabeceira da cadeira, para evitar a compressão dos vasos sanguíneos (Santos \& Pereira, 2020).

Porém, muitos problemas relacionados à cavidade bucal de gestantes poderiam ser evitados com cuidados básicos como orientações sobre higiene bucal e aplicação tópica de flúor, que podem ser feitos em qualquer época da gestação, sem ofertar nenhum perigo ao feto (Faria, 2010). 


\subsection{Alterações bucais}

A gestação é acompanhada por diversas mudanças fisiológicas na mulher, necessitando assim de cuidados especiais temporários. Quando falamos da saúde bucal, o equilíbrio dela pode ser alterada por diversos motivos durante esse período, uma vez que a mulher estará mais sujeita a alterações hormonais, a uma dieta não balanceada, enjoos regulares e dificuldade de realizar a higienização bucal (Silva, Couto, \& Conceição, 2020).

As principais alterações bucais que ocorrem durante o período gestacional são a cárie, gengivite e periodontite. No entanto, essas alterações não ocorreriam isolamente apenas pelo fato da gravidez, mas sim por estarem atrelados a negligência na escovação e nos hábitos alimentares, como a maior ingestão de açúcar (Sampaio, 2019).

Já a cárie, pode vir a ocorrer por motivos hormonais, aumento do estrôgeno; alterações no pH salivar, redução da concentração de sódio, tornando o meio favoravél ao agravo; e também devido a gengivite, que pode, devido aos enjoos, tornar a escovação e os cuidados rotineiros menos agradáveis, acentuando o desenvolvimento da cárie (Hemalatha et al., 2013).

A gengivite é considerada por alguns autores como uma alteração normal durante a gravidez. Isso pode ser explicadas pela alteração hormonal que em níveis elevados, aumenta a permeabilidade vascular, gerando uma hiperplasia gengival, acentuando a resposta gengival frente ao biofilme dentário (Hemalatha, Manigandan, Sarumathi, Aarthi, \& Amudhan, 2013).

A gengivite gravídica, na maioria das vezes começa no segundo mês de gestação e sua principal característica é uma resposta exacerbada do organismo a mínima presença de biofilme, devido aos altos níveis de hormônios. Clinicamente a gengiva apresenta sangramento ao simples toque da escova durante a escovação, edema e uma cor avermelhada. Contudo, é de extrema importância um controle adequado, utilizando recursos como: escovação, fio dental e bochechos, sendo a higiene um fator determinante para o tratamento (Oliveira, Lopes, Santos, \& Magalhães, 2014).

A periodontite, uma infecção cronológica, causada em decorrência da presença em excesso de bactérias anaeróbicas que atingem o osso alveolar, cemento e o ligamento periodontal, podendo apresentar-se de forma severa, caracterizada por ligeira progressão para o sangue, podendo afetar o bebê e a placenta. Como consequência da periodontite, alguns autores apontam a relação da dela com o elevado risco de partos prematuros (Puertas et al., 2018).

Vários fatores podem influenciar o surgimento da doença, como a deficiência de higiene, doenças sistêmicas, como é o caso da diabetes, e a própria gestação, visto que nesse período, o organismo da mulher sofre muitas alterações morfológicas. Pois as gestantes estão expostas a altas concentrações de hormônios esteroides produzidos pela placenta. Esses possuem receptores no tecido gengival, acumulando-se o que constitui um importante fator para o crescimento bacteriano (Dourado, 2018).

A doença periodontal está associada também com a pré-eclâmpsia, que é pressão alta acima de 140/90 mmHg, acima de 20 semanas. No entanto ainda são necessários que mais trabalhos sobre esse tema sejam feitos. Embora os estudos mostrem a associação da doença com problemas que possam a vir ter na gestação, não se sabe ao certo que realizando o tratamento as pacientes ficarão livres de complicações obstétricas. Até o momento, sabe-se que onde há uma população com baixo índice de partos prematuros e bebês abaixo do peso a terapia periodontal não mostrou resultado no desfecho. Já em populações onde essas complicações são acima de 20\%, há indícios que a terapia periodontal possa diminuir o risco, porém a depender do nível (Duque, 2013).

\subsection{Anestésicos locais}

Quando os profissionais entrevistados foram questionados sobre qual anestésico local de escolha para as pacientes gestantes. Levantamentos de Cechinel et al. (2016); Rodrigues et al. (2018); Prado et al. (2019); Bernardi et al. (2019) demonstraram que a lidocaína foi o anestésico mais apontado pelos cirurgiões-dentistas. Porém, mesmo que em percentuais 
baixos, os estudos de Rodrigues et al. (2018) (1,60\%), Prado et al. (2019) (9\%), Bernardi et al. (2019) (2,4\%), alguns profissionais elegeram a prilocaína como o anestésico de primeira escolha.

Para Navarro et al. (2008), a prilocaína deve ser evitada pela possibilidade de gerar contrações uterinas devido à sua apresentação no Brasil associada ao vasoconstrictor Felipressina - substância similar com o hormônio responsável por contrações uterinas durante o trabalho de parto. Prado et al. (2019) contraindicam a prilocaína devido a facilidade em atravesar a placenta e efeito metehemoglobinizante no feto. Silvia e Sanchez (2017) também corroboram que a prilocaína não deve ser indicada.

No levantamento realizado por Caneppele et al. (2011), o sal anestésico mais votado $(14,33 \%)$ pelos profissionais foi a mepivacaína, No entanto, Prado et al. (2019) em seu estudo afirma que esse anestésico deve ser evitado não apenas durante a gestação, mas também durante o período de amamentação por conta do recém-nascido não metabolizar bem este anestésico no fígado. Bernardi et al. (2019), acrescentam ainda o risco da mepivacaína causar bradicardia fetal.

Com relação ao vasoconstrictor e a sua concentração, divergências também foram encontradas nos trabalhos analisados. Segundo Lee, Milgrom, Huebner e Conrad (2010), a epinefrina na concentração de 1:100.000 deve ser o vasoconstrictor de escolha, não devendo ultrapassar o limite de dois tubetes por sessão. Já para Caneppele et al. (2011), Silva e Sanchez (2017), o máximo de tubetes por sessão não deve ser superior a três.

\subsection{Prescrição medicamentosa}

É de extrema importância levar em consideração os possíveis riscos e benefícios da indicação de qualquer substância terapêutica na gravidez, principalmente durante o primeiro trimestre de gestação, pois neste período ocorre a organogênese, fase em que os órgãos do feto estão se desenvolvendo, sendo considerado um período crítico para a suscetibilidade teratogênica. Já no período fetal $\left(60^{\circ}\right.$ dia até o final da gestação), período de crescimento e aperfeiçoamento das funções, alguns medicamentos também podem determinar alterações em certos órgãos (Olivo, 2013).

Quanto ao analgésico de primeira escolha, a pesquisa de Prado et al. (2019) divergiu com os estudos de Rodrigues et al. (2018); Bernardi et al. (2019) e Caneppele et al. (2011), pois a dipirona foi apontada com o maior percentual (57\%). O paracetamol foi indicado como primeira escolha e a dipirona como segunda opção, pois esta pode profocar agranulocitose, redução do número de granulócitos no sangue (neutropenia), ficando a gestante mais susceptível a infecções (Moreira, Santin, Matos, Gravida, \& Faquim, 2015; Rodrigues et al., 2018).

Entre os dos anti-inflamatórios, o ibuprofeno foi eleito por alguns profissionais como uma alternativa, dentres eles nos estudos de Caneppele et al. (2011) (2,5\%), Rodrigues et al. (2018) (9,30\%), Prado et al. (2019) (14\%). Já no trabalho de Bernardi et al. (2019), 29,3\% prescrevem o anti-inflamatório sem receios; 34,1\% concordam parcialmente com esta conduta; 9,8\% dos profissionais discordam do entendimento de que o uso desse medicamento seja seguro, e $22 \%$ nunca os utilizariam.

O ibuprofeno, analgésico e anti-inflamatório não esteroidal, deve ser prescrito com cautela em pacientes grávidas, uma vez que esse medicamento pode causar alterações na circulação sanguínea fetal. Além disso, inibem a síntese de prostaglandinas, podendo ocasionar o fechamento do ducto arterial e como consequência a hipertensão pulmonar e morte do feto (Nogueira, 2018).

Com relação a classe dos antibióticos, a penicilina foi votada como primeira escolha nos estudos de Caneppele et al. (2011) (65\%), Rodrigues et al. (2018) (75,20\%), Prado et al. (2019) (79\%) e Bernardi et al. (2019) (91,70\%), sendo a amoxicilina a mais indicada entre dos grupos de antibióticos. As penicilinas são os antimicrobianos de primeira escolha quando quadros de infecções sistêmicas são apresentados durante a gestação, por apresentarem ação bactericida e baixa toxicidade, não causando danos a gestante e nem ao feto (Brasil, 2010). Já o metronidazol não deve ser administrado durante a gestação e nem lactação, pois é tido como potencialmente teratogênico (Wannmacher, 2015). 


\subsection{Flúor}

A indicação do uso do flúor tem sido um assunto de muito debate nos últimos anos, pois seu uso estava relacionado à proteção do dente do futuro bebê. Mas se sabe que o flúor administrado durante a gestação não tem nenhum efeito preventivo no bebê. Todavia, não se recomenta a aplicação sistêmica de flúor durante a gestação, pois se sabe que o flúor tem participação no processo de remineralização dos elementos dentários, aumentando a quantidade de fluorapatita na superfície do esmalte dentário, e não na sua formação (Oliveira \& Gonçalves, 2009).

A suplementação pré-natal não e indicada principalmente por meio de complexos vitamínicos que tenham cálcio, pois este reage com o flúor diminuindo sua absorção. A sua diminuição não traz nenhum prejuízo, mas a de cálcio sim, pois este elemento é de extrema importância para a paciente gestante e para o bebê. Alguns autores afirmam que apesar de médicos obstetras prescreverem suplementos com flúor essa conduta não tem nenhuma comprovação cientifica, já que o flúor administrado durante a gestação não oferece proteção contra cárie do bebê. Contudo, não foi relatado nenhum problema relacionado a ingestão de flúor durante a gestação (Oliveira \& Gonçalves, 2009).

\subsection{Exames radiográficos}

Quanto as radiografias, as pesquisas mostraram como os cirurgiões-dentistas divergem quanto a este procedimento. $\mathrm{Na}$ pesquisa de Caneppele et al. (2011), quando questionados, 66,5\% realizavam se as vantagens superassem claramente os riscos e com uso de avental de chumbo e 32,4\% não realizavam em gestantes, já com relação ao trimestre, 56,7\% evitam tomadas radiográficas somente no primeiro trimestre, $3,2 \%$ no último trimestre e $40,1 \%$ não fazem radiografias em gestantes. No estudo de Prado et al. (2019), 64\% submetem as pacientes gestantes a tomadas radiográficas se as vantagens destas superarem claramente os riscos e com a utilização do avental de chumbo e $20 \%$ submeteriam nas mesmas condições que sem a proteção do avental.

As radiografias odontológicas não são contraindicadas para pacientes gestantes, uma vez que a exposição à níveis de radiação são muito baixos, tornando pequeno o risco de efeitos nocivos. Como auxílio de diagnóstico em odontologia, as radiografias devem ser realizadas sempre que necessária, no entanto a proteção com avental de chumbo na região do abdômen e pescoço da gestante não deve ser negligenciada como medida de proteção (Ebrahim, Oliveira, Peres, \& Franco, 2014).

\subsection{Atenção básica na gravidez}

É durante a gestação a melhor época de orientar as mulheres sobre higiene bucal, a necessidade de controle do biofilme dentário e também de uma alimentação adequada, pois é nesse momento que elas estão mais propensas a adquirir novos conhecimentos, visando o bem estar da própria gestante e de seu futuro bebê, que podem levar a aquisição de novos hábitos saudáveis permanentes (Matsubara, 2017; Ribeiro, 2019).

Visando isso, o Sistema Único de Saúde (SUS), através da Estratégia da Saúde da Família (ESF), vem organizando Programas Nacionais de atenção à saúde da mulher, incluindo gestantes, por saber da importância em ofertar um serviço de reabilitação e recuperação da saúde bucal. Assim, faz parte da ESF realizar a assistência as gestantes de sua área, realizando o pré-natal (Lampert \& Bavaresco, 2017).

O pré-natal tem como objetivo acolher a mulher em todo o período gestacional, garantindo que desde o momento que a mulher tem conhecimento da sua gravidez até o nascimento saudável da criança e a garantia do bem-estar materno e o neonatal. Nesse sentido, a orientação é a captação o quanto antes das gestantes com a realização da primeira consulta pré-natal em até 120 dias de gestação, sendo realizadas, no mínimo, seis consultas, preferencialmente, uma no primeiro trimestre, duas no segundo trimestre e três no último trimestre da gestação (Brasil, 2011). 
Dentro dos serviços prestados pelo pré-natal, inclui o odontológico. Durante a gravidez, a mulher fica temporariamente em risco a alguns problemas bucais, devido as alterações fisiológicas que podem ocorrer nesse período. Dessa forma, o pré-natal odontológico é de suma importância, visto que se torna uma medida de promoção da saúde, contribuindo não somente com a estabilidade da saúde bucal, como também na prevenção de futuros problemas sistêmicos (Nogueira, 2018).

Durante o pré-natal odontológico, a gestante é orientada sobre os cuidados com a higiene e alimentação, sendo feito também um exame clínico dos dentes e tecidos moles, a partir daí, é elaborado um plano de tratamento e os procedimentos odontológicos indicados são executados ao longo da gestação. A Política Nacional de Saúde Bucal (Brasil Sorridente) aconselha ainda a relevância de se desenvolver ações educativo-preventivas. Uma vez que a mãe é considerada como papel fundamental nos padrões de comportamento adquiridos pela criança durante a primeira infância (Brasil, 2004).

Assim, com a concepção e confirmação da gravidez, a mulher, para realizar o pré-natal odontológico, deve ser encaminhada à equipe de saúde bucal (ESB), com finalidade de garantir a consulta avaliativa e continuidade de acordo com a necessidade individual de cada paciente (Brasil, 2004).

\section{Considerações Finais}

É importante que o cirurgião-dentista detenha conhecimento e esteja preparado para que o atendimento às gestantes seja realizado de forma adequada. Estas pacientes podem ser atendidas em qualquer trimestre de gestação, sendo o segundo, o mais seguro e confortável. O exame radiográfico pode ser realizado como auxílio diagnóstico, sempre com avental de chumbo, levando em consideração que a dose recebida pela mãe em uma radiografia dentária é muito inferior àquela que pode causar má formação congênita. A fluorterapia pode ser realizada de forma tópica apenas para melhora da saúde bucal da gestante. É muito importante que o profissional analise os principais sinais vitais da gestante, principalmente a pressão arterial. Todo procedimento passível de dor, deve ser precedido por anestesia local também nas gestantes e se não houver reais complicações médicas, deve-se optar pela lidocaína a $2 \%$ com epinefrina 1:100.00. No que se trata de terapêutica medicamentosa, o antibiótico de eleição é penicilina e paracetamol, o analgésico de primeira escolha, deve-se evitar antiinflamatórios nãoesteróidais, e quando extremamente necessário, usa-se corticoides. A posição mais indicada para a gestante na cadeira odontológica é colocando-a voltada para o lado esquerdo para evitar compressão da veia cava inferior. A maioria dos estudos mostrou que, apesar de muitos profissionais afirmarem sentirem-se seguros em atender pacientes grávidas, isso não garantiu que alguns, respondessem adequadamente aos questionamentos. O que reforça a importância da inclusão do tema no currículo de graduação em odontologia.

Por fim, vale ressaltar, que mesmo sendo um tema de bastante importância para a área odontológica e da saúde, o conteúdo abordado ainda se mostra escasso nas pesquisas. Por isso, esse trabalho ratifica a necessidade de que mais trabalhos sejam realizados com a finalidade de difundir os protocolos adotados durante a gravidez, e que assim os tratamentos sejam feitos de formas precisas e seguras, trazendo benefícios para o profissional, para gestante e para o bebê.

\section{Referências}

Assunção, A. C. \& Miguel, D. A. (2015). Assistência odontológica a gestante e lactante com ênfase no tratamento endodôntico (Trabalho de conclusão de curso). Instituto de Estudos da Saúde Sérgio Feitosa, Belo Horizonte, http://iesposgraduacao.com.br/assets/downloads/2dceb3de5166919254d1d9154cb8f8d6.pdf

Bastiani, C., Cota, A. L. S., Provenzano, M. G. A., Fracasso, M. L. C., Honório, H. M. \& Rios, D. (2010). Conhecimento das ge stantes sobre alterações bucais e tratamento odontológico durante a gravidez. Odontologia Clínico-Científica, 9 (2), 155-160. http://revodonto.bvsalud.org/scielo.php?pid=S167738882010000200013\&script=sci_arttext.

Bernardi, C., Oliveira, J. B. \& Masiero, A. V. (2019). Assistência odontológica à gestante: conhecimento e prática de dentistas da rede pública e seu papel na rede cegonha. Arq. Odontol., 55. https://periodicos.ufmg.br/index.php/arquivosemodontologia/article/view/12557/13111. 
Brasil, Ministério da Saúde. (2004). Secretaria de Atenção à Saúde. Departamento de Atenção Básica. Coordenação Nacional de Saúde Bucal. Diretrizes da Política Nacional de Saúde Bucal. Brasília: Ministério da Saúde. http://bvsms.saude.gov.br/bvs/publicacoes/politica_nacional_brasil_sorridente.htm.
Brasil,
Ministério
da Saúde
(2010)
Formulário
terapêutico
nacional
$(2 a$
ed.),

https://bvsms.saude.gov.br/bvs/publicacoes/formulario_terapeutico_nacional_2010.

Brasil, Ministério da Saúde. (2011). Portaria MS/GM n. 1.459. Institui, no âmbito do Sistema Único de Saúde - SUS - a Rede Cegonha. http://bvsms.saude.gov.br/bvs/saudelegis/gm/2011/prt1459_24_06_2011.html.

Caneppele, T. M. F., Yamamoto, E. C., Souza, A. C., Valera, M. C. \& Araújo, M. A. M. (2011). Conhecimento dos cirurgiões-dentistas sobre o atendimento de pacientes especiais: hipertensos, diabéticos e gestantes. Journal of Biodentistry and Biomaterials, 1, 31-41. http://www.unibjournal.com.br/pdf/revista1artigo4.pdf.

Cechinel, D. B., Boff, W. M., Ceretta, R. A., Simões, P. W., Ceretta, L. B. \& Sônego, F. G. F. (2016). Sistematização de um protocolo de atendimento clínico odontológico a gestantes em um município sul catarinense. Rev. Odontol. Univ. Cid. São Paulo, 28(1), 6-16. http://arquivos.cruzeirodosuleducacional.edu.br/principal/old/revista_odontologia/pdf/janeiro-abril_2016/Odonto_01_2016_6-16.pdf.

Dourado, B. M. R. (2018). Doença periodontal em gestantes e repercussões gestacionais e ao recém-nascido (Dissertação de mestrado). Universidade Estadual Paulista Júlio de Mesquita Filho, Botucatu, SP, Brasil. https://repositorio.unesp.br/handle/11449/155852.

Duque, C. (2013). Odontopediatria: uma visão contemporânea. Santos.

Ebrahim, Z. F., Oliveira, M. C. Q., Peres, M. P. S. M. \& Franco, J. B. (2014). Tratamento odontológico em gestantes. Science in Health, 5 (1), $32-44$ http://arquivos.cruzeirodosuleducacional.edu.br/principal/new/revista_scienceinhealth/13_jan_abr_2014/Science_05_01_32-44.pdf.

Faria, D. M. F. (2010). Atendimento odontológico às gestantes (Trabalho de conclusão de curso). Universidade Federal de Minas Gerais, Contagem, MG, Brasil. 2010. https://repositorio.ufmg.br/bitstream/1843/BUBD-9KDQG5/1/conclus_o_da_monografia.pdf.

Hemalatha, V. T., Manigandan, T., Sarumathi, T., Aarthi, N. \& Amudhan, A. Dental Considerations in Pregnancy - A critical review on the oral care. Jounal of Clinical and Diagnostic Research, 7 (5), 948-953. https://www.ncbi.nlm.nih.gov/pmc/articles/PMC3681080/pdf/jcdr-7-948.pdf.

Lampert, L. \& Bavaresco, C. S. Atendimento Odontológico à gestante na atenção primária. RSC online, 6 (1), 81-95. https://rsc.revistas.ufcg.edu.br/index.php/rsc/article/view/169/165.

Lee, R. S., Milgrom, P., Huebner, C. E. \& Conrad, D. A. (2010). Dentists' perceptions of barriers to providing dental care to pregnant women. Womens Health Issues, 20 (5), 359-365. https://pubmed.ncbi.nlm.nih.gov/20800772/.

Martinelli, K. G., Belotti, L., Poletto, Y. M., Neto, E. T. S. \& Oliveira, A. E. (2020). Fatores associados ao cuidado de saú de bucal durante a gravidez. Arq. Odontol., 56. https://periodicos.ufmg.br/index.php/arquivosemodontologia/article/view/16353/19301.

Matsubara, A.S. \& Demetrio, A. T. W.; Atendimento odontológico às gestantes: Revisão de literatura. Revista Uningá Review, 29 (2). http://revista.uninga.br/index.php/uningareviews/article/view/1959.

Moreira, M. R., Santin, G. C., Matos, L. G., Gravida, D. B. L. \& Faquim, J. P. S. (2015). Pré-natal odontológico: noções de interesse. Journal of Management \&Primary Health Care, 6 (1), 77-85. http://jmphc.com.br/jmphc/article/view/234.

Moretti, A. S., Garcia, V. B., Cruz, M. C. C. da, Rolim, V. C. de B. \& Sakashita, M. S. (2018). P oo9 - A importância do atendimento odontológico em gestantes. Archives of Health Investigation, 6. https://archhealthinvestigation.com.br/ArcHI/article/view/2824.

Navarro, P. S. L., Dezan, C. C., Melo, F. J., Alves-Souza, R. A., Sturion, L. \& Fernandes, K. B. P. (2008). Prescrição de medicamentos e ane stesia local para gestantes: conduta de cirurgiões-dentistas de Londrina, PR, Brasil. Revista da Faculdade de Odontologia de Porto Alegre, 49 (2), 22-27. https://seer.ufrgs.br/RevistadaFaculdadeOdontologia/article/view/3039/8284.

Nogueira, P. M. (2018). O cuidado odontológico à gestante na rede pública de atenção primária de Belo Horizonte (Dissertação de Mestrado). Universidade Federal de Minas Gerais, Belo Horizonte, MG, Brasil. https://repositorio.ufmg.br/handle/1843/ODON-B37K3P.

Oliveira, E. C., Lopes, J. M. O., Santos, P. C. F. \& Magalhães, S. R. (2014). Atendimento odontológico a gestantes: A importância do conhecimento da saúde bucal. Revista de Iniciação Científica da Universidade Vale do Rio Verde, $4 \quad$ 1), http://periodicos.unincor.br/index.php/iniciacaocientifica/article/viewFile/1550/1210.

Oliveira, J. F. M. \& Goncalves, P. E. (2009). Verdades e mitos sobre atendimento odontológico da paciente gestante. Revista Portuguesa de Estomatologia, Medicina Dentaria e cirurgia Maxilofacial, 50 (3), 165-171. https://www.sciencedirect.com/science/article/pii/S1646289009701189.

Olivo, S. M. (2013). Atendimento odontológico a gestantes: Mitos e Preconceitos por parte dos Cirurgiões Dentistas (Trabalho de Conclusão de curso). Universidade Federal de Santa Catarina, Brasil. https://repositorio.ufsc.br/bitstream/handle/123456789/105870/tccsarahmenghelolivofinal.pdf?sequence=1.

Prado, L., Nunes, L. M. S., Figueiredo, R. L., Silva, R. B. V., Cerdeira, C. D. \& Santos, G. B. (2019). Conduta de cirurgiões-dentistas no atendimento à paciente gestante. Revista Científica da UNIFENAS, 3(1), 18-28. http://revistas.unifenas.br/index.php/revistaunifenas/article/view/269.

Puertas, A., Magan-Fernandez, A., Blanc, V., Revelles, L., O’Valle, F., Pozo, E., León, R. \& Mesa, F. (2018). Association of periodontitis with preterm birth and low birth weight: a comprehensive review. J. Matern Fetal Neonatal Med., 31 (5), 597-602. https://pubmed.ncbi.nlm.nih.gov/28282773/.

Ribeiro, L. P. (2019). Assistência odontológica na gestação (Trabalho de Conclusão de Curso). Centro Universitário do Planalto Central Apparecido dos Santos - UNICEPLAC, Brasília, DF, Brasil. https://dspace.uniceplac.edu.br/handle/123456789/243. 
Research, Society and Development, v. 10, n. 1, e24510111701, 2021

(CC BY 4.0) | ISSN 2525-3409 | DOI: http://dx.doi.org/10.33448/rsd-v10i1.11701

Rodrigues, G. L., Nogueira, P. M., Fonseca, I. O. M., Ferreira, R. C., Zina, L. G. \& Vasconcelos, M. (2018). Pré-natal odontológico: assistência às gestantes na rede pública de atenção básica em saúde. Arquivos em Odontologia, 54. https://periodicos.ufmg.br/index.php/arquivosemodontologia/article/view/3754.

Rother, E. T. (2007). Revisão sistemática X revisão narrativa. Acta Paulista de Enfermagem, 20(2), 5-6. https://www.redalyc.org/pdf/3070/307026613004.pdf.

Sampaio, E. B. (2019). Percepção de gestantes acerca da saúde bucal na gravidez (Dissertação de Mestrado). Universidade Federal do Ceará, Fortaleza, CE, Brasil. http://repositorio.ufc.br/handle/riufc/49376.

Santos, G.C. \& Pereira, D. P. C. (2020). A importância da odontologia no cuidado da gestante: Revisão de literatura. Revista Multidisciplinar e de Psicologia. 14 (50), 1212-1230. https://idonline.emnuvens.com.br/id/article/view/2530.

Silva, L. G., Couto, L. S. \& Conceição, L. S. (2020). Cuidados odontológicos no pré-natal. Facit Business and Technology Journal, 2 (16), 174-180. http://revistas.faculdadefacit.edu.br/index.php/JNT/article/view/616/462.

Silva, M. E. A. \& Sanchez, H. F. (2017). Proposta de protocolo clínico para atendimento odontológico a gestantes na atenção à saúde. Revista de APS, 20 (4), 628-635. https://periodicos.ufjf.br/index.php/aps/article/view/15652.

Vasconcelos, R. G., Vasconcelos, M. G., Mafra, R. P., Júnior, L. C. A., Queiroz, L. M. G. \& Barboza, C. A. G. (2012). Atendimento odontológico a pacientes gestantes: como proceder com segurança. Rev. Bras. Odontol. 69(1), 120-124. http://revodonto.bvsalud.org/scielo.php?script=sci_arttext\&pid=S003472722012000100027 .

Wannmacher, L. \& Ferreira, M. B. C. (2012). Farmacologia Clínica para Dentistas (3a ed.) Guanabara Koogan. 